\title{
La tradición académica occidental en el estudio ecosistémico: el Lago de Pátzcuaro como un ejemplo de ineficacia y racismo epistémico
}

\author{
Western academic tradition in ecosystem study: \\ Lake Pátzcuaro as an example of inefficiency and epistemic racismo
}

Fernando Bernal Brooks*

Resumen: El presente caso de estudio ecosistémico centrado en el lago de Pátzcuaro trata aspectos -ambientales, políticos, económicos y sociales- entrelazados-. La versión científica de la realidad desde el paradigma positivista sobrevalora los "datos duros" obtenidos en instalaciones frecuentemente apartadas del contexto histórico y social de las comunidades indígenas. La ganancia en "objetividad" desde una visión de "expertos" lleva implícito el costo de la inefectividad práctica para revertir el impacto humano sobre el ambiente lacustre.

Palabras clave: México, Pátzcuaro, epistemicidio, racismo, indígena.

\begin{abstract}
The present ecosystemic case study centered on lake Patzcuaro deals with environmental, political, economic, and social- interlinked aspects. The scientific version of reality from the positivist paradigm overvalues the "hard data" obtained in facilities far from the historical and social context of indigenous communities. The gain in "objectivity" from an "experts" view, bears implicit the cost of practical inefficacy to revert the human impact on the lacustrine environment.
\end{abstract}

Keywords: Mexico, Pátzcuaro, epistemicide, racism, indigenous.

Recibido: 12 noviembre 2020 Aceptado: 4 enero 2021

\section{Introducción}

Desde las divagaciones en un escritorio aislado y un racismo epistémico impuesto que anula la otredad/sujeto subalterno indígena, Smith (1999) reconoce en el término "investigación" una asociación inextricable al colonialismo y al imperialismo europeos. En contraparte, las otras formas de conocer y producir conocimiento (Denzin y Lincoln, 2011) y, en particular, la investigación indigenista, considera a las personas indígenas "como el conjunto de aquellos que fueron testigos, excluidos y sobrevivientes de la modernidad y el imperialismo. Para esta orientación, la historia de las investigaciones está, por una parte, estrechamente enlazada con la colonización y con su reproducción y, por otra, apartada de la posibilidad de desarrollo y autodeterminación de los pueblos indígenas.”. Por

\footnotetext{
* Mexicano. Profesor-Investigador de la Universidad Michoacana de San Nicolás de Hidalgo con el auspicio del Consejo Nacional de Ciencia y Tecnología para el proyecto "Jardín Etnobiológico Purhépecha Juchari Uinapikua" con vigencia entre 2020 y 2021. E-mail: fernando.bernal@umich.mx
} 


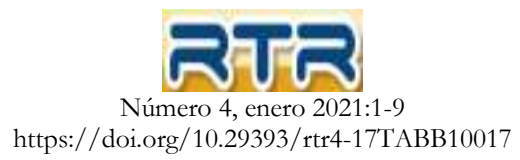

ende, el paradigma decolonial busca desmantelar los dispositivos ideológicos de la colonialidad del poder, cuya disolución de estructuras de dominación y explotación, conlleva hoy en día en el conocimiento académico, una propuesta epistémica, teórica y metodológica que pretende superar esta (injusta y racista) matriz histórica (GESCO, 2012).

El presente artículo pone a consideración del lector el caso del lago de Pátzcuaro, un tema que entremezcla aspectos -ambientales, 'políticos, sociales y económicos-, el cual cuenta con una investigación realizada por décadas desde una aproximación positivista con una tríada ineficaz en la solución de problemas ambientales ecosistémicos: "expertos o especialistas" (normalmente académicos), "no expertos" (gente del pueblo con conocimientos supuestamente elementales por el hecho de no haber ido a la escuela), al igual que los tomadores de decisiones en la esfera gubernamental que a su vez ponen en práctica el manejo del ambiente lacustre con base en la opinión de "expertos".

$\mathrm{Al}$ respecto surgen las siguientes interrogantes: ¿De qué ha servido emprender investigaciones sobre el entorno lacustre de Pátzcuaro si la búsqueda de soluciones a los problemas ambientales que aquejan a la población aledaña no arroja resultados palpables? Desde una visión fragmentaria procedente de las universidades (bosques, agua, biodiversidad, etc., cada aspecto por su lado) y sin la participación de la gente en el proceso mismo de la investigación: ¿Cuál es hasta el momento la contribución de la academia desde el paradigma occidental dominante cuando aquellos que deben poner en práctica las medidas o sugerencias para remediar o restaurar el ambiente quedan en un plano secundario como "no expertos"; aún a pesar del contacto ancestral con el medio natural y los saberes obtenidos de generación en generación? La pasividad resultante, al igual que sucede en los salones de clase donde se practica una educación bancaria (sensu Paulo Freire) contribuye a generar espectadores inmóviles con la fe puesta en gente estudiada, pero nuevamente volvemos a preguntar ¿Cuál es la significancia y el beneficio para las comunidades indígenas purhepechas (quienes deben recibir los beneficios de la investigación denominada "científica") de la manera supuestamente idónea de aprehender la realidad existente y transformarla? A continuación, el contraste entre los saberes indígenas y las consecuencias de las opiniones de "expertos".

\section{Ecología de saberes}

Argueta y Castilleja (2018) advierten "no hemos encontrado que los cronistas o autores posteriores señalen que los colonizadores (españoles) les enseñaron a los purhepechas a pescar, ni mucho menos a cultivar peces". Obviamente, la sabiduría ancestral que proviene de la interacción cotidiana con los recursos naturales se transmite a través de la tradición oral -medio de comunicación en las comunidades-; sin embargo, no produce documentos ni es susceptible de aprendizaje en los muros de una escuela. Lo anterior, por lo tanto, no encuadra en los círculos académicos diseñados por una sociedad occidental para dar validez al conocimiento.

Por citar un ejemplo, la denominación indígena de las especies de peces existentes en el lago de Pátzcuaro: Kurucha urapiti (significa pescado blanco en idioma P'urhepecha) carece de validez científica al igual que los nombres locales de las demás especies existentes (detalles en Argueta y Castilleja, 2018), no así aquellos nombres designados por expertos desde fuera del país y en latín, en este caso Menidia estor. La imposición desde fuera del nombre designado en la lengua muerta europea resulta incomprensible para las comunidades Purhepechas y discrimina epistemológicamente, la forma local de generación del saber sobre los recursos naturales.

En palabras de Argueta y Castilleja (2018): "el conocimiento colectivo sobre los peces del lago implica no solo su identificación como especies distintas y las relaciones entre ellas, sino también el reconocimiento detallado de las diversas formas en cómo se alimentan, qué comen y como lo hacen, también sobre dónde habitan (al interior del lago) y en dónde y a qué profundidad, y por todo ello, 
cuando es el mejor tiempo y lugar para su captura. También conocen la forma en que se aparean para la reproducción y si el cuidado de las crías lo hace la hembra o el macho, si les gusta nadar solos o en grupos, si en noche de luna llena nadan en la profundidad y si en la luna nueva lo hacen en la superficie".

En la actualidad, el proyecto denominado "Jardín Etnobiológico Purhépecha Juchari Uinapikua" busca visibilizar los saberes ancestrales y la investigación-acción participativa desde el involucramiento activo de las comunidades ribereñas en el lago de Pátzcuaro.

\section{La opinión de "expertos" y las medidas de manejo puestas en práctica por el gobierno}

\section{Introducción de especies}

Una de las operaciones más imprudentes emprendidas por parte del gobierno federal radica en la importación del lirio acuático (Eicchornia crassipes) en 1898 (Miranda y Lot, 1999), el cual apareció a Pátzcuaro alrededor de 1907 aproximadamente. La región de Pátzcuaro depende del turismo como fuente de ingresos de tal manera que el transporte de pasajeros a la isla de Janitzio constituye una de las actividades económicas importantes en la región. Así que, la proliferación excesiva de las plantas flotantes de dicha especie no solo obstruye la navegación sino desluce estéticamente el panorama lacustre. Por ende, en 1960 inicia el combate frontal al indeseable lirio mediante la adquisición de una draga tipo "Grijalva" para su extracción mecánica (La Voz de Michoacán, 30 de abril, 1960).

Por iniciativa del gobierno federal a cargo del general Lázaro Cárdenas del Río, el black bass norteamericano (lobina negra o trucha como se conoce en Pátzcuaro) ingresó a las aguas del lago de Pátzcuaro, con el objetivo fallido de impulsar la actividad turística. La consecuencia de tal decisión llevada a efecto en 1936 (Dosil, 2018) afectó en gran medida a las especies nativas ya existentes al arribar en el entorno lacustre un voraz depredador que encontró a su disposición una buena cantidad de organismos nativos de tamaño adecuado para alimentarse (De Buen, 1941; García de León, 1984; Toledo, 1995).

En 1969, la Secretaría de Hacienda y Crédito Público emprende "el desarrollo de la piscicultura mediante la cría y siembra de la carpa de Israel y lobina, señalando que las comunidades beneficiadas serán Chupícuaro y Santa Fe de la Laguna” (La Voz de Michoacán, 17 de junio de 1969). Rosas (1976) comenta que "después de esta negativa introducción de la lobina negra que depreda y compite con las valiosas especies nativas, se han hecho otras introducciones: de carpa herbívora (Ctenopharingodon idellus, como solución verde a la proliferación de lirio acuático) en 1972, de Tilapia melanopleura en 1974 y, de carpa de Israel (Cyprinus carpio), de esta última se ignora la fecha y cantidad introducida".

Durante el régimen presidencial de Luis Echeverría Álvarez (1970-1976), el Fideicomiso para el Desarrollo de la Fauna Acuática (FIDEFA) a través de la Estación Limnológica de Pátzcuaro impulsó la transfaunación de especies de un lado al otro en el país. "Directamente de las acciones acuaculturales, proceden la mayoría absoluta de las especies introducidas, a las que se podrían agregar las de control biológico (del lirio acuático, por lo regular) y las accidentales, ya que las primeras deben ser cultivadas y las segundas, que aumentan con una frecuencia alarmante, son especies que llegan confundidas entre las que son intencionalmente introducidas y que reflejan el desconocimiento de los encargados de controlar su distribución en los centros de producción" (Contreras Balderas, 1999).

Es así como desde la sede en Pátzcuaro inicia una mezcla de especies sin precedente en las aguas interiores nacionales y, por citar un ejemplo, aparece el pescado blanco de Chapala (Menidia lucius) en Pátzcuaro con la consecuente hibridación documentada con la especie local (Alaye, 1993). Ahora, "para enfrentar el deterioro genético de las poblaciones de pescado blanco, algunos autores han sugerido 


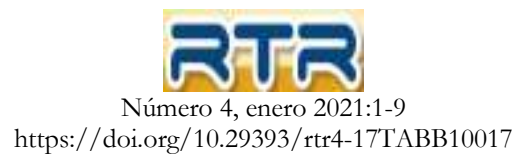

como prioritario, al igual de lo que se ha hecho con otras especies, la formación de un banco de genoma, involucrando al mayor número posible de centros de investigación y dependencias gubernamentales" (Dosil, 2018).

Más aún, la introducción de especies de peces desde Pátzcuaro hacia otras regiones incluye, en el caso de las carpas chinas, la diseminación a la par de los parásitos de peces procedentes del país asiático.

Hacia finales de los años 1970s, "en el colmo de las soluciones improvisadas y carentes de reflexión ambiental (especialmente para controlar el lirio acuático), se importaron de las aguas tibias de Chiapas... un par de manatíes (hembra y macho) con la peregrina idea de que incluso se reprodujeran en las frías aguas del Lago de Pátzcuaro" (Argueta y Castilleja, 2018).

Con el cambio de condiciones en el lago (escasa profundidad y mayor turbidez), el estudio más reciente sobre "las especies exóticas del lago de Pátzcuaro" (Zambrano et al. 2011) resta importancia al black bass y a la carpa herbívora; más bien centra la atención en la carpa común y la tilapia como nuevos protagonistas en el escenario lacustre deteriorado. En otras palabras, el cuerpo de agua sufrió transformaciones progresivas que dieron lugar a un ecosistema lacustre totalmente distinto al que conocieron las comunidades ribereñas purhepechas.

\section{Introducción de redes de monofilamento}

Hasta finales de los años 1960s, la pesca dependía de artefactos elaborados con paños de hilos de algodón. Ciertamente, después de cada faena, el arte de pesca utilizado debía ser secado al sol para evitar la degradación. Con la introducción de las fibras sintéticas en el mercado, las redes de monofilamento superaron en eficacia a los métodos anteriores por la ventaja de permanecer dentro del agua indefinidamente y este hecho en particular elevó considerablemente el esfuerzo de captura de especies y la presión de la pesca en el ecosistema lacustre.

\section{Adquisición de maquinaria}

Como ya se mencionó anteriormente, el control mecánico del lirio inicia con la adquisición de una draga tipo "Grijalva" en 1960. Ante el fracaso de introducir en el lago de Pátzcuaro la carpa hervíbora y los manatíes para el control del lirio acuático, el gobierno federal retomó la compra de maquinaria a principios de los años 1980s para acometer la indeseable proliferación de la vegetación acuático y así también retirar los "azolves" del lago. Como antecedente a tales acciones, la teoría evolutiva del Dr. de Buen (1943) sostenía que el lago sufre de la acumulación de sedimentos procedentes de una periferia deforestada (por lo tanto habría que retirar tales azolves o acumulaciones de sedimentos del lago) y de la consecuente eutroficación que conlleva el ingreso de agroquímicos en las aguas del lago desde los campos agrícolas circunvecinos, lo que a su vez facilita la aparición de la "maleza" acuática sobre la zona ribereña.

El nombre "maleza", en particular, alude al problema que ocasiona la presencia de vegetación acuática en el agua (en especial el lirio) en tanto obstruye la navegación de las embarcaciones; en este caso, las lanchas comerciales que cruzan el trayecto del embarcadero principal de Pátzcuaro hacia la isla de Janitzio. La derrama económica que origina el turismo justificó tales inversiones que eventualmente asumió el gobierno del estado de Michoacán mediante un Programa de Dragado fijo a cargo de la Comisión de Pesca del gobierno del estado.

Sin duda, una de las preocupaciones más importantes de principios de los 1980s para empezar a escarbar en la ribera del lago centró la atención en tres puntos de la zona sur: 1) El embarcadero principal de Pátzcuaro, 2) San Pedro Pareo, con la finalidad de "marcar la raya" a los agricultores que aprovechaban el descenso de nivel del lago ya que facilitaba la apropiación de terrenos antes inundados con aguas del lago (una contradicción con la teoría evolutiva centrada en los azolves). A la par, el producto 


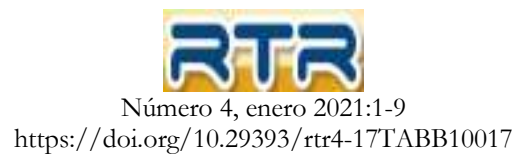

del desazolve permitió desarrollar un sistema de chinampas (a semejanza de Xochimilco) sobre la zona federal en beneficio del CONALEP y la novedosa carrera de técnico en producción acuícola vigente en aquellos momentos. 3) El conjunto de islas de Urandén en la preocupación de preservar los "usos y costumbres" indígenas como un ambiente cercado por agua donde las prácticas de canotaje adquieren trascendencia internacional.

\section{Plantas de tratamiento de agua}

De las varias obras construidas para tratar el agua residual en los municipios colindantes al lago, las únicas instalaciones que operan a la fecha se localizan en el muelle de San Pedrito, junto a la Tsipekua. La inversión millonaria inicia con un proyecto concebido desde la Secretaría de Desarrollo Urbano (SEDUE) y parte de la decisión gubernamental de contener el vertimiento de aguas negras y la consecuente proliferación del lirio acuático en el lago. Como un asunto competencia de "expertos", la ejecución de la obra carece del respaldo social activo y, muy probablemente, hasta del conocimiento de muchos en la región.

La eventual cesión de la citada planta de tratamiento de agua al gobierno municipal ocurre sin la contratación debida del personal especializado y sin el presupuesto operativo correspondiente, de tal manera que la prioridad para los habitantes de la región en cuanto a obras de mayor relevancia social (caminos, escuelas, agua potable, etc.) dejó dichas instalaciones sin posibilidades de operar a su máxima eficiencia. De parte del organismo operador del agua potable, el restablecimiento de la calidad del agua después del uso doméstico o industrial en los alrededores de Pátzcuaro, no aparece en su agenda como un tema importante.

\section{Instalación de presas de gavión o piedra acomodada}

En la óptica de "un lago medio lleno de sedimentos", nuevamente, habría que hacer algo por contener los sedimentos en el mismo lugar donde se generan, en la contraparte terrestre. De ahí surge la idea de represar el producto de la erosión antes de su llegada al lago en la parte terrestre.

\section{Acuacultura y cooptación de las comunidades}

El Fideicomiso para el Desarrollo de la Fauna Acuática (1972-1976) operó con base en la contratación de pescadores del lago de Pátzcuaro como personal técnico, dada la experiencia ancestral de los Purhepechas en la pesca lacustre. Este hecho facilitó el adiestramiento de nuevos pescadores en distintos lugares del país para la captura de especies de peces -desde la elaboración misma de las artes de pesca a partir de materias primas-, como una ocupación tendiente no solo a generar empleo e ingresos, sino como una fuente de diseminación de especies por la prioridad que adquirió la acuacultura extensiva durante la época.

Como parte de los saberes locales, "la observación reiterada de los sitios de desove del pez blanco dio lugar a que en San Andrés Ziróndaro y en Santa Fe de la Laguna, aunque posiblemente también en San Jerónimo y otros lugares del lago, se desarrollara una técnica para apoyar y proteger el desove natural que cada año ocurría y ocurre en la zona norte del lago" (Argueta y Castilleja, 2018). El impulso a esta actividad acuícola a principios de los 1980s desde la Secretaría de Pesca y su delegación en Michoacán configuró un Programa de Reserva de Especies Nativas con la participación conjunta entre el gobierno federal y los pescadores del lago. ¿En qué consistía? La elaboración de jaulas flotantes facilitaba la incubación de huevos fecundados de pescado blanco y acúmara para la posterior liberación de crías en el medio acuático a determinada talla que garantizara la sobrevivencia de los organismos a etapas adultas. 


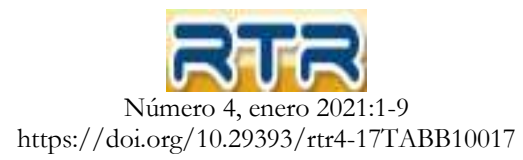

No obstante, el gobierno federal perdió funciones operativas importantes con la denominada "federalización" (fortalecimiento de las entidades) a principios de los años 1980s y aún más, por las consecuencias del terremoto de 1985. Por tanto, las delegaciones federales de Pesca cedieron obligaciones a los gobiernos estatales en una política pública que da lugar a la creación de la Comisión de Pesca del gobierno del estado de Michoacán. Desde ahí, la manipulación del programa de especies nativas en Pátzcuaro por funcionarios del gobierno del Estado con fines electoreros (y con el apoyo de "expertos") condujo eventualmente a la cooptación y al favoritismo por un solo grupo apostado en una de las islas de Urandén, actualmente conocido como la Reserva de Urandén. Aun cuando la producción pesquera del lago jamás repuntó, el sitio figura como el escaparate para reportar a la ciudadanía la siembra por millones de unidades de las dos especies mencionadas en el párrafo anterior, mientras los funcionarios estatales y municipales aparecen en las fotos para el periódico.

\section{Vedas y decomisos de artes de pesca}

"Hacia el 18 de febrero de 2000, la SEMARNAP emite el aviso por el establecimiento de una veda temporal para la captura de todas las especies de peces y anfibios en la jurisdicción federal del lago de Pátzcuaro... (desde la visión de los "expertos") sin ser consultado e informado a los interesados, (lo que) dio origen al decomiso de redes por parte de la PROFEPA y al drama del encarcelamiento y la condena, injusta y desmesurada, de 20 años de prisión en contra de..." (Argueta y Castilleja, 2018) dos personas y órdenes de aprehensión para otras dos, lo que ocasionó una fuerte crisis y la ruptura de las relaciones entre el gobierno y las comunidades del lago con resentimientos entre la gente que perduran a la fecha.

\section{Antecedentes de la influencia gubernamental sobre la movilización social}

En un intento por propiciar la movilización social asistida desde los agentes externos, el sexenio gubernamental de Ernesto Zedillo (1994-2000) a través de la SEMARNAP (conducida por Dra. Julia Carabias) contempló una visión holística e integral en el manejo de los recursos naturales, con la Comisión Nacional del Agua como parte de su superestructura. En Pátzcuaro, la conformación de un Comité para el manejo del lago logra un hecho sin precedente: la participación e interacción de los diferentes gremios involucrados en la problemática ambiental del lago, como son: agricultura, forestal, pesca, sector educativo, etc.

En el sexenio de Vicente Fox (2000-2006) y en adelante, la entusiasta participación ciudadana en Pátzcuaro impulsada desde una política pública nacional aminoró posteriormente tras un vuelco hacia las empresas (neoliberal) que otorgó mayor importancia a la producción en una modalidad de "producir por producir" sin importar el impacto sobre el hábitat de las especies ni las consecuencias ambientales. Nuevamente, la obtención de conocimientos sobre el lago quedó supeditada a los recursos asignados a una instancia de "expertos", particularmente el Instituto Mexicano de Tecnología del Agua (IMTA). El financiamiento adicional por parte de la fundación Gonzalo Río Arronte al "Programa para la Recuperación Ambiental de la cuenca del lago de Pátzcuaro" facilitó que personal de dicha institución realizara visitas periódicas hasta la fecha desde las instalaciones situadas a la distancia, en Cuernavaca, Morelos.

\section{Reflexiones finales}




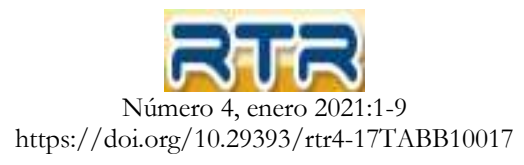

El presente documento muestra los resultados de la implementación de medidas ambientales basadas en la investigación positivista del lago de Pátzcuaro, con un largo proceso de estudio a partir de 1939 (De Buen, 1941, 1943, 1944a, 1944b); el cual ha generado datos y más datos ajenos al posible significado para las comunidades indígenas Purhepechas (receptoras del posible beneficio); es decir, un racismo epistemológico combinado con el culto a la personalidad de figuras académicas (Bernal.Brooks, 2017) que, en suma, restan "objetividad" al proceso mismo de la investigación.

Para Boaventura de Souza Santos (2011), el epistemicidio ocurre con la destrucción de saberes propios de los pueblos causada por el colonialismo europeo y norteamericano (europeos desplazados) perpetrado desde las universidades y centros de investigación en concurrencia con un "pensamiento colonizado" en la mayoría de los habitantes (en este caso México) que data de la invasión española del siglo XVI; lo que facilita sobreponer y privilegiar la visión occidentalizada y eurocéntrica del conocimiento sobre los saberes locales ancestrales.

Sin duda, el estudio del lago de Pátzcuaro desde el positivismo arremete contra la cosmovisión Purhepecha y socava el principio: "Juchari Eratsekua" (nuestra forma de pensamiento). El autor del presente documento sostiene que las comunidades purhepechas no precisan de una labor asistencialista por parte del gobierno o las universidades, más bien el sector local requiere definir con quien y como deberá operar la investigación desde el rol activo de la gente. Tampoco es indispensable la participación de un "experto" que elabore un proyecto "de escritorio" desde alguna instancia ajena a la comunidad, sino que el proceso mismo de la investigación empodere a las comunidades en la consecución de sus objetivos propuestos desde la horizontalidad. "El proceso decolonizador que viene ganando espacio en la investigación se involucra en luchas múltiples que tienen lugar en emplazamientos diversos. Implica la deconstrucción o desarmado del imperialismo y del colonialismo en sus formas antiguas y nuevas, junto al reclamo por un conocimiento, un lenguaje y una cultura, así como la búsqueda de soberanía y transformación social de las relaciones coloniales entre el nativo y el colonizador" (Smith, 2011)... cuya continuidad en México ocurre bajo los auspicios de las grandes mayorías que discriminan a las comunidades indígenas tal cual lo aprendieron a partir de la invasión española en el siglo XVI.

En consecuencia, el sector indígena, que vive en las inmediaciones del lago de Pátzcuaro, resulta el más afectado en la imposición de ideas por "expertos" y proyectos surgidos del exterior para resolver la problemática local. Las fallidas medidas de manejo así lo demuestran. Luego entonces, ¿De qué manera contribuye el conocimiento científico en revertir el deterioro ambiental del lago? Más allá de los abundantes documentos puramente descriptivos sobre la parte estructural del ecosistema (variables físicas y químicas del cuerpo de agua o las existencias de plantas o animales que existen en el lugar), las aportaciones teóricas en cuanto al funcionamiento del ecosistema no rebasan el nivel de dos hipótesis aun a pesar de la existencia de una cantidad formidable de datos disponibles que han encumbrado a Pátzcuaro como "uno de los lagos más estudiados de América Media" y más "estudiados en el continente en todos sus aspectos"-. (Deevey, 1957).

Una reflexión sencilla sobre las aportaciones "científicas" pone de manifiesto lo siguiente: ¿Cuál es la relevancia de definir en términos cuantitativos el nivel de clorofila $a$ en las aguas del lago (en ocasiones más de 200 microgramos por litro) como un indicador de la fertilidad del agua si de antemano la simple observación y el sentido común dejan ver un problema perceptible de contaminación? (Es obvio que lo tiene que decir un "experto" desde la academia). Por un lado, los números así obtenidos reflejan un estado de "congelamiento" fotográfico para un lugar y momento determinados; si el caso de estudio cuenta con una red de muestreo suficiente en el sentido espacial y temporal del caso, la esperanza del investigador estriba en armar un mosaico coherente de momentos fotográficos que a su vez faciliten la interpretación y la elaboración de un relato de "expertos" acerca del lago. Al final del camino, la versión "cuantitativa" de los hechos no va más allá de lo que "salta a la vista”. 


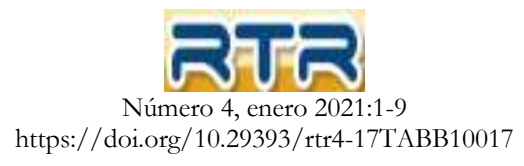

Más aun, las recomendaciones prácticas derivadas de dicho conocimiento suelen aparecer desligadas de los datos en sí y más bien atisban en una sección al final de los documentos de manera espontánea y tangencial. El investigador, por lo general, sugiere medidas de remediación ya conocidas ampliamente con antelación (tecnología, sobre todo) o recurre a la apropiación de los saberes locales de la gente -en este caso el pueblo indígena purépecha que habita la ribera del lago ancestralmente- para justificarse y dar explicaciones. Esto significa que los argumentos desde la perspectiva positivista alcanzan la envestidura de "aportaciones científicas", no tanto por la aplicación de una metodología rigurosa, sino por una autoridad moral que alguien con el prestigio en el medio académico valida e impone en su carácter de "experto" sobre otros "no expertos". Algo similar ocurre entre medicina tradicional vs. medicina alopática.

El hecho palpable es que toda la investigación realizada en torno al lago de Pátzcuaro resulta poco trascendente desde la manera tradicional de cómo llevarla a efecto para resolver los problemas del lago, ni tampoco desde el para qué pues no responde a las necesidades de las comunidades; más bien contribuye a enaltecer el ego de aquel que investiga desde su posición de privilegio. Aún más grave, el ir y venir de los investigadores desde sus sedes en universidades o instituciones de gobierno, adolece de una falta de compromiso social genuino sobre las consecuencias de sus recomendaciones (al igual que un médico alópata trata con clientes, no con seres humanos; o un arquitecto que elude la responsabilidad cuando los edificios se caen tras un terremoto) y, sin duda, todas las decisiones de manejo que se han puesto en práctica en el lago de Pátzcuaro en complicidad con autoridades del gobierno soslayan la responsabilidad, sin lugar a dudas, de dejar dañado el ecosistema lacustre en el afán primordial de enaltecer el prestigio social de los "expertos" y cobrar comisiones en complicidad. Utilizar la bandera de la neutralidad política de la ciencia positivista resulta, por tanto, un buen negocio. Finalmente, Dosil (2018) comenta de manera atinada: "En definitiva, se trata de generar una conciencia ciudadana que apueste por un verdadero desarrollo sustentable, lo cual nunca podrá lograrse sin una modificación de nuestros hábitos y sin un reparto equitativo de las responsabilidades: los maltrechos pescadores no pueden (como los campesinos en otros espacios) padecer siempre los desaciertos de un modelo de sociedad insostenible.

\section{Referencias}

Alaye, N. (1993). El pescado blanco (género Chirostoma) del lago de Pátzcuaro, Michoacán. Composición de especies. Ciencia Pesquera 9.

Argueta, A. y Castilleja, A. (2018). Conocimientos y tecnología p'urhepecha sobre la pesca en el lago de Pátzcuaro. En: Florescano, E. y Sánchez, G. (coordinadores) El Pescado Blanco en la Historia, la Ciencia y la Cultura Michoacana. Morelia: Secretaría de Cultura.

Bernal Brooks, F. W. (2017). La objetividad de las ciencias naturales: un meta-análisis limnológico y psicológico de las investigaciones sobre el lago de Pátzcuaro. Teoría y Crítica de la Psicología 9: 98-123.

Contreras-Balderas, S. (1999). Acuacultura, ictiodiversidad, transfaunación acuática y peces en riesgo en México. Memorias de la IV Reunión Nacional de Redes de Investigación en Acuacultura. Cuernavaca, Mor. 19-21 Octubre de 1999.

De Buen, F. (1941). El Micropterus (Huro) salmoides y los resultados de su aclimatación en el lago de Pátzcuaro. Revista de la Sociedad Mexicana de Historia Natural 2: 69-78.

De Buen, F. (1943). Los lagos michoacanos. I. Caracteres generales. El lago de Zirahuén. Revista de la Sociedad Mexicana de Historia Natural 3-4.

De Buen, F. (1944a). Los lagos michoacanos. II. Pátzcuaro. Revista de la Sociedad Mexicana de Historia Natural 1-2. 
De Buen, F. (1944b). Limnobiología de Pátzcuaro. Anales del Instituto de Biología (México) 15

De Souza Santos, B. (2011). "Epistemologías del Sur". Utopía y Praxis Latinoamericana, 54, 17-39.

Deevey, E. S. Jr. (1957). Limnologic studies in Middle America with a chapter on aztec limnology. Transactions of the Connecticut Academy of Arts and Sciences 39.

Denzin, N. K. y Lincoln, Y. S. (2011). Manual de Investigación Cualitativa. El campo de la Investigación Cualitativa. Volumen I. Barcelona: Gedisa.

Dosil, J. (2018). El pescado blanco en la encrucijada. Una mirada desde la historia de las ciencias. En: Florescano, E. y Sánchez, G. (coordinadores) El Pescado Blanco en la Historia, la Ciencia y la Cultura Michoacana. Morelia: Secretaría de Cultura.

García de León, F. J. (1984). Ecología pesquera, alimentación y ciclo gonádico de Chirostoma estor Jordan y Micropterus salmoides Lacépéde, en el lago de Pátzcuaro, Michoacán. México. Tesis de Licenciatura. Universidad Autónoma de Nuevo León.

Grupo De Estudios Sobre Colonialidad (GESCO). (2012). Estudios Decoloniales: Un panorama general. KULA: Antropólogos del Atlántico Sur (6), pp. 8-21.

Miranda, M. G. y Lot, A. (1999). El lirio acuático ¿una planta nativa de México? Ciencias 53: 5054.

Rosas, M. (1976). Datos biológicos de la ictiofauna del lago de Pátzcuaro, con especial énfasis en la alimentación de sus especies. En Memorias del Simposio de Pesquerías en Aguas Continentales. Ciudad de México: Secretaría de Industria y Comercio.

Smith, L. T. (1999). Decolonizing Methodologies: Research and Indigenous Peoples. Dunedin, NZ: University of Otage Press.

Smith, L. T. (2011). Caminando sobre terreno resbaladizo. La investigación de los pueblos nativos en la era de la incertidumbre. En Denzin, N. K. y Lincoln, Y. S. Manual de Investigación Cualitativa. Vol. I. El campo de la investigación cualitativa.

Toledo Díaz-Rubín, P. (1995). Consumo de aterínidos (Chirostoma spp) por la lobina negra (Micropterus salmoides) en el lago de Pátzcuaro, Mich. Ciencia Pesquera 11: 71-74.

Zambrano, L., Córdova Tapia, F., Ramírez Herrejón, J. P., Mar Silva, V., Bustamante, L., Camargo, T., y Bustamante, E. (2011). Las especies exóticas en el lago de Pátzcuaro, Michoacán, México. En Huerto Delgadillo, R., Vargas Velázquez, S., y Ortíz Paniagua, C. (eds.) Estudio Ecosistémico del lago de Pátzcuaro. Instituto Mexicano de Tecnología del Agua. 